\title{
Customizing the Human-Computer Interface to Compensate for Individual Cognitive Attitude: An Exploratory Study
}

\author{
Sylnovie Merchant \\ California State University, Sacramento, CA, USA
}

smerchant@esus.edu

Abstract

A major concern facing system developers is how well the system will operate for the intended user. The aspect which allows a user to interact the system is referred to as the Human-Computer Interface (HCI). This paper discusses the various approaches advocated by researchers in an attempt to explore the issues surrounding HCI.

Keywords: human-computer interface, user, system development

\section{Introduction}

System developers face many challenges and decisions as they analyze and create designs. Among the many factors to be considered is how the system will function and operate for the intended user. The aspect of the system that allows the user interaction with data, software, or hardware is typically called the Human-Computer Interface, or HCI (Dewitz, 1996). For many years researchers have debated whether individual characteristics such as cognitive styles, personality, traits, and or cultural differences should be considered as a basis for customized system design at the HCI. In addition, the notion of "improving" the user interface has become a frequent theme in popular computer literature as users have expressed their dissatisfaction with design.

Jefferson (2000) postulates that the two main reasons for continued "lousy interfaces" are the reduction in the importance of end-user experience for the sake of developing future growth and the idea that developers design based on the requirement of the operating system rather than for the end-user. Manes (2000) describes some of the ongoing problems with common interfaces which anger and confuse users. His article represents the growing public sentiment of interface dissatisfaction and the desire for customization. While many valid arguments have been made that support customization from both academic research and users themselves, efforts to employ the necessary methodologies required to implement them on a broad level has been slow.

The purpose of this research is to help determine whether design customization based on individual cognitive differences at the HCI is warranted or justified. This topic is of particular significance because users play an important part in the execution of the information system and its successful ability to meet the goals of the organization.

Material published as part of these proceedings, either on-line or in print, is copyrighted by Informing Science. Permission to make digital or paper copy of part or all of these works for personal or classroom use is granted without fee provided that the copies are not made or distributed for profit or commercial advantage AND that copies 1) bear this notice in full and 2) give the full citation on the first page. It is permissible to abstract these works so long as credit is given. To copy in all other cases or to republish or to post on a server or to redistribute to lists requires specific permission from the publisher at publister@intormingscience.org

\section{Human-Computer Interface ( $\mathrm{HCl})$}

There are various definitions of the HumanComputer Interface (HCI). Each definition provides a somewhat different perspective of the discipline as well as insight into the factors that comprise it. Two generally accepted definitions have been gen- 
erated by both professional groups and scholars. The Curriculum Development Group of the Association for Computing Machinery (ACM) Special Interest Group on Computer-Human Interaction (SIGCHI) defines it as a discipline concerned with the design, evaluation, and implementation of interactive computing systems for human use and with the study of major phenomena surrounding them. Another definition comes from a leading HCI textbook which defines HCI as the study of people, computer technology, and the ways they influence each other. The text further states that the study of HCI is necessary in order to determine how we can make computer technology usable by people (Head, 1999). Even when simply put these definitions reveal that the study of HCI involves a number of complex and varied systems between and among the computer and the user. Because of these factors, the study of HCI is largely interdisciplinary. Contributions to the study come from computer science, engineering, cognitive science, and social science, to name a few (Carroll, 1997; Dix, et al, 1998).

The interface can be further described by the way it communicates. This is sometimes referred to as the design language. Design language is made up of three primary parts. The first of these three parts refers to design elements. These elements consist of icons, color, and other screen functions. The second part of the design language is the organizing principles. These are generally rules that govern how design elements will be used in order to convey meaning to the user. Finally qualifying principles consider the context for opportunities, such as multimedia capabilities, and the limitations, such as software variability (Head, 1999). The more tangible aspects of the user interface usually consist of a screen, what is presented on the screen (e.g. icons and menus), and what we use to communicate with the screen (e.g. a mouse, touch, or pen).

When developers are creating a design they make decisions about what elements to use, how to present them to the user and how they will function. The design of the HCI is crucial to achieving the goals for the new system because it facilitates understandability, acceptance, learning, use, and satisfaction for the user (Karat, et al, 2000). The HCI is of particular importance to developers because users play a critical role in the execution of the information system and its successful ability to meet the goals and objectives of the organization that it serves.

\section{Background}

At the inception of HCI research, users were not at all considered in the development of computer systems and software. Designing for the needs, abilities, and preferences of users was not taken seriously. According to Carroll (1997) most of the writings about computing from the mid-1970's were dismissive of usability and patronizing of users. HCI truly began to evolve when the typical user shifted from engineers and programmers to non-technical individuals (Grudin, 1990).

The original foundations of HCI were based largely in cognitive theory, which focused primarily on learning, error and error recovery, preference fatigue, and individual differences (Dix, 1998). Cognition is a large field of study. It can be defined as processes that involve acquiring, storing, and using knowledge. It includes aspects of attention, perception, learning, memory, and problem solving. This cognitive approach has lead to another related field, cognitive ergonomics, which can be described as the interaction between human information processing capabilities and limitations and technical information processing systems (Waern, 1989).

Since this time research has been conducted on many factors that ultimately contribute to the design of the HCI. Some factors considered are associated with the computer and its elements, such as equipment (e.g. screen type and size), speed (e.g. processor type) and, programming (e.g. software utilization). While other factors considered are associated with the user, such as training, experience, and individual differences. More attention to the HCI revealed a need for further research regarding the influence of the user on system objectives. 
Early research on the HCI identified potential key contributors to individual differences such as cognitive style, personality measures, and demographic or situational variables (Zmud, 1979). In the mid 1980's a body of specific research had begun to develop which focused around individual cognitive differences and how they impact the HCI (Neal, 1987).

\section{Empirical Research on the Human-Computer Interface and Cognition}

Research studies were attempting to narrow various cognitive characteristics and were creating design solutions that might enhance the HCI (Benyon and Murray, 1993). Research on spatial memory and design alternatives in text editing revealed performance for individuals, who scored low for spatial memory, increased greatly when using a screen-based editor (which was intended to reduce spatial memory demands) compared with a line editor (Gomez, et al, 1983). Differences were also discovered when cognitive factors, such as strategy selection and recall of operational details, were measured in terms of task performance and accuracy. In this case subjects were tested with both a dedicated physical control system and one that employed the use of desired icons, pop-up menus, and a touch panel. A 77 percent difference in enhanced performance was attributed to differences in the subject's cognitive makeup (Bailey, et al, 1988).

More recently, Loshe (1997) found that differences in working memory, for tasks of high complexity, were attributed to increased accuracy when subjects were given two graphic decision aids. These results supported the hypothesis that design decisions regarding graphs and other visual aids reduce cognitive load by shifting to the visual perception system. Westerman (1997) also examined implications regarding cognitive styles and the HCI. This research revealed that users who were categorized as "experts" in computer ability and who additionally scored low on cognitive spatial ability, performed most similar to novices with high spatial ability.

It is important to note that during the late eighties and early nineties research involving the HCI, cognitive factors, and customization fell off sharply. This may have been due to the fact that traditional research was unable to keep pace with rapidly expanding and evolving technology. The industry entered a period where the focus was on conforming the user to system applications and not the reverse. At this point industry attention was directed more toward training strategies (Carroll, 1997). More recently there has been a resurgence of research intended to identify individual cognitive factors and use them in the application of the HCI. This interest has generated from the infusion of newer technologies, which have advanced sciences of artificial intelligence and virtual reality, both of which are inexplicitly grounded in research in human cognition (Carroll, 1997).

\section{Current Research on the $\mathrm{HCl}$, Cognition, and Design Factors}

Research in this area has taken a more deliberate step in the direction of design alternatives. Newer research has attempted to promote the practical application of cognitive theories on design strategies. Because of the promotion of HCI specialist into the realm of project management, research has continued to evolve from lab-based theories and results to usable strategies (Carroll, 1997).

One example is as technology has advanced, changes in screen design and communication tools have evolved. Data are now presented on a variety of screen sizes and types. Small screen sizes, which are used with many new telecommunication tools, such as wireless Internet access cellular phones, are expected to gain enormously in popularity. Rahman and Muter (1999) examined the cognitive variables of reading efficiency and preference when users were given four types of small display presentations. Reading efficiency was defined as reading speed and comprehension. In the study, conditions were manipulated in order to determine under what circumstances a small display window might be used without compromising user performance. In the first condition four conditions were tested. Subjects read from a computer screen in a sentence-by-sentence presentation which the subjects pressed a button to advance the prompt. 
The results indicated that sentence-by-sentence reading was just as efficient as normal page reading. In the second experiment, the sentence display was set at varying rates. This condition revealed that performance was still achieved due to the reduction of eye movements within the reading space depending on the condition.

Another recent study by Gray (2000) attempted to find the nature and processing of errors in interactive behavior. This study examined key-press errors when using devises with the approximate complexity of a VCR. The study attempted to elucidate the relationship between VCR use and devices with similar interface strategies that are used in emergency rooms, airplane cockpits, and power plants. Although many key-presses were correct the paper examined the subcategories and types of errors. It also examined the ultimate impact of the key-press errors on the completion and overall success of the task goal. It was determined that further user training could reduce errors of ignorance. However, errors of "skilled" users were significantly reduced when cognitive engineering was applied to the design of the device. Another significant discovery was that errors made on four percent of the VCR key-presses would have been responsible for two-thirds of the failure to complete the task (recording a show).

There are several implications for this study with regard to cognitive design. One is the ratio of errors to the percentage of the completion of the task. This may imply that small computing errors may be responsible for many of the reasons that tasks are not completed. For example, using the mouse manipulate an icon which unintentionally ends up in an unintended spot. Another example is a key-press error that results in the accidental deletion of a document.

These studies and many others strongly suggest that in general, individual differences in cognition have a significant impact on user performance with regard to design alternatives.

\section{Applying Cognitive Factors to the $\mathrm{HCl}$ Design}

The HCI field of HCI has been generally considered fragmented due to the variety of methodologies and theoretical constructs that have developed from its multi-disciplinary nature (Carroll, 1997). This has made it difficult for developers to assimilate and apply relevant research findings in a real-world setting. The results of these findings leave developers with some important questions. How can customization be applied to design alternatives on a larger scale rather than a case-by-case basis? The following articles propose ways of identifying and integrating user factors into the HCI.

User Centered Design, or UCD, was created with the intent to provide developers with a comprehensive guide to customization. Kramer et al (2000) proposed a six step UCD design process. The first step is to determine the user's wants and needs. This process generally involves obtaining a great deal of information from the intended user. For example, ideally it includes obtaining the breadth of their technical knowledge. Obtaining personalization requirements is key to UCD therefore the authors suggest that a better way to obtain this type of information is to exchange the technical design for a market analysis of these factors. This would entail using a set of profile elements, which are feasible to acquire, and working solely within those parameters. Second, is the use of standard task analysis methods. These are used in order to learn the impetus for users' actions, methods of completing the task, and the ultimate intention of the user. Goal trees and activity flows are then used to design through needs-based recommendations. These are intended to provide a road map to trace the importance of a task and determine its value to the user. The third step involves obtaining the ultimate desirable set of functions for the user. This is done without regard to real-world constraints, which enables creativity of design, and is used for the creation of novel task flows. According to the authors the key to mass customization requires the use of ObjectOriented, or OO, design. At step four OO analysis and documentation is developed. The fifth step combines all of the previous steps into an end-to-end analysis of each potential user experience and documenting using interactions diagrams. Finally, the accessibility for each object (and ultimately what the user sees) is constructed using visual treatment rules. 
Another stride toward standardizing customization is from machine-learning algorithms that are intended to learn the patterns and stereotypical sequences of a user's actions, essentially tracking cognitive flow. Ideally, the appropriate "self-customizing software" application would recognize how the system is being used and enhance user performance. There are two types of systems that lend technology to this premise. The first is content-based prediction. This technology bases predictions for user preferences on the content of the artifacts that have been used. For example, News Dude uses this technology to determine the news-stories of interest to its users. It has a reported seventy-five percent accuracy in predictions. The Incremental Probabilistic Action Modeling (IPAM) software was designed to predict the future needs of the user by accounting for the history of past actions in a user profile. According to the authors, the IPAM has a reported forty percent accuracy. By combining content-based methods and IPAM methods into a collaborative method the authors predict that customization with a high degree of accuracy can be attained.

Greenberg (1998) suggests presents a more simplistic method. The author suggests using a standardized guide of cognitive theories in order to design interfaces. Designers are specifically directed to focus attention on understanding the cognitive principles of visual perception, limits of memory, attention span, and spatial elements. Many of these methods are currently considered in HCI design texts.

Each of these articles presents challenges and alternatives for developers when considering cognition in the HCI. However, design methodologies still fall short of a comprehensive guide for developers.

\section{Considerations for Designing the $\mathrm{HCl}$}

There are still questions that must be answered with regard to design practices. Given these findings what direction should developers take when designing the HCI? What cognitive factors should be considered in the design? How will developers decide which factors are important to use and under what circumstances?

These questions reveal a need for basic research to identify the cognitive factors, which are best suited to different types of interfaces. The best example provided is the work done by Rahman and Muter (1999) on small display windows and reading comprehension. The authors used a growing instrument design (the small window display) and identified the main objectives for optimal use. By manipulating the display readout (the independent variable) and measuring elements of comprehension (the dependent variable) they were able to make design application recommendations. These types of studies on cognition are essential to the body of knowledge necessary to create customized designs that provide the user with the greatest utility.

These questions also reveal the need for continued and more clearly defined academic research that also provides relevance in a "real-world" setting. It is important that the research reflect both the benefit scientific empiricism, as well as, an adherence to relevant and practical application. Problems with relevance have been addressed in many fields of computer study (Benbasat and Zmud, 1999).

Many valid arguments have been made in favor of customization but circumstances have slowed progress. One of the reasons progress in this area has been slow is due to the multi-disciplinary nature of the HCI. Many contributions from numerous studies have overwhelmed the literature with experiments, which explore the details but provide little overall direction.

Another contributing factor is the fact that technology has been changing so rapidly that some research has lacked relevance. By the time development, funding, approval, and research has been conducted the variables under investigation have changed or become obsolete. Technological advances have also put the needs of users in the proverbial back seat. Sentiment for meeting the desires of users seem to come and go depending on the types of new advancements available. 
A third reason progress has been slowed by a lack of relevance that is attributed to differences between laboratory conditions and application to the "real-world". Although solutions for these situations are being remedied, it has left many developers waiting for solutions and advancements in this area.

\section{Conclusions}

Users play an important role with regard to the successful execution of a system and their interaction has become a primary focus of research and development. This interaction, the HCI is comprised of many factors that contribute to the users ability to use the intended system. User cognition is one of those factors. Public sentiment supports the notion that customization on varying levels is essential to user satisfaction. Empirical research supports the hypotheses that individual differences in cognition contribute to variance in the ultimate accomplishment of goals intended by system developers. Researchers believe that customizing aspects of the HCI will enable better success in this area. More current research into the areas of artificial intelligence and virtual reality has produced beneficial paradigms with regard to customization and cognition.

Three important factors to consider are the impact that rapidly changing technology has on the application of a theory, the difference between controlled circumstances which are constructed in a laboratory setting and the "real-world", and the problems with focusing a multi-disciplinary field into widely applicable solutions. In addition, it is important for developers to keep asking the questions which will stimulate the research needed to facilitate a better HCI which allows customization for its users cognitive needs.

Some researchers have attempted to provide an applicable and standardized form for developers to use as a guide for customization practices. These methodologies fall short in some crucial areas. They are still in a developmental stage that is unable to facilitate a wide application. However, the studies examined here do provide a guide and direction in which to formulated ideas. These studies also provide a measure of achievement in the area of customization in which developers should look to for advancement in the future.

Developers must have a standard in order to determine what cognitive variables need to be applied and under what circumstances to use them. These conditions have tended to raise more questions than provide solutions.

\section{Recommendations}

There are three areas that will help advance the efforts to provide developers with the necessary tools to assess and design interfaces that facilitate individual cognition. First, developers should fully utilize academic institutions by gaining their support for relevant and timely projects. With access from developers and business organizations studies could be conducted within the "real-world" setting instead of environments which do not provide a representative arena.

Secondly, business organizations and users should become more vocal about the problems they experience at the interface level. Instead of spending resources attempting to train and retrain users to conform to a poorly designed interface, efforts could be directed toward educating users about cognitive elements in order to work with developers on design ideas. There are thousand of users that may be a potential unending source of inspiration and ideas.

Finally, both educators and developers need to stay focused on the user, and the circumstances in which systems fail at the interface level, in order to further understand and develop ideas and solutions.

\section{References}

Bailey, W. A., Knox, S. T., \& Lynch, E. F. (1988). Effects of Interface Design Upon User Productivity Interface Evaluations.

Proceedings of ACM CHI'88 Conference on Human Factors in Computing Systems 1988 Abstracts, $207-212$. 
Benbasat, I., \& Zmud, R. W. (1999). Empirical Research in Information Systems: The Practice of Relevance. MIS Quarterly, $23(1)$, pp. 3-16.

Benyon, D., \& Muray, D. (1993). Developing Adaptive Systems to Fit Individual Aptitudes Session 4: Adaptive Systems. Proceedings of the 1993 International Workshop on Intelligent User Interfaces 1993 Abstracts, 115-121.

Carroll, J. M. (1997). Human-computer interaction: Psychology as a science of design. Annual Review of Psychology, 48, p. 61.

Dewitz, S. (1996). Systems Analysis and Design and the Transistion to Objects. San Francisco: Irwin/McGraw-Hill

Dix, A. J., Finlay, J. E., Abowd, G. D., \& Beale, R. (1998). Human-Computer Interaction. New York: Prentice Hall.

Gomez, L. M., Egan, D. E., Wheeler, E. A., Sharman, D. K., \& Gruchacz, A. M. (1983). How Interface Design Determines Who Has Difficulty Learning To Use a Text Editor. Text Editors Proceedings of ACM CHI'83 Conference on Human Factors in Computing Systems 1983 Abstracts, 176-181.

Gray, W. D. (2000). The Nature and Processing of Errors in Interactive Behavior. Cognitive Science, 2000, 2, p. 205.

Greenberg, C. J. (1998). Beyond HTML! Information Outlook, 2 (11), p 26.

Grudin, J. (1990). The Computer Reaches Out: The Historical Continuity of Interface Design Evolution and Practice in User Interface Engineering. Proceedings of ACM CHI'90 Conference on Human Factors in Computing Systems 1990 Abstracts, 261-268.

Head, A. J. (1999). Design Wise. Medford, New Jersey: Information Today, Inc.

Hirsh, H, Basu, C., \& Davidson, B. D. (2000). Learning to Personalize. Association of Computing Machinery, Communications of the ACM, New York, 43 (8), pp. 102-106.

Jefferson, S. (2000). Beyond the keyboard and mouse. Infoworld, 22 (44), p 51

Karat, J., Karat, C., \& Ukelson, J. (2000). Affordances, motivation, and the design of user interfaces. Communications of the ACM, $43(8), \mathrm{p} 49$.

Kramer, J., Noronha, S. \& Vergo, J. (2000). A user-centered design approach to personalization. Association of Computing Machinery, Communications of the ACM, New York, 43 (8), pp 44-48.

Lohse, G. L. (1997). The Role of Working Memory on Graphical Information Processing Design and Evaluation of User Interface Software. Behaviour and Information Technology Abstracts, 16 (6), 297-308.

Manes, S. (2000). The curse of user-hostile design. PC World, 18 (11), p 284.

Neal, L. (1987). Cognition-Sensitive Design and User Modeling for Syntax-Directed Editors Adaptive Interfaces. Proceedings of ACM CHI+GI'87 Conference on Human Factors in Computing Systems and Graphics Interface 1987 Abstracts, 99-102.

Waern, Y. (1989). Cognitive Aspects of Computer Supported Tasks. New York: John Wiley \& Sons.

Westerman, S. J. (1997). Individual differences in the use of command line and menu computer intefaces. Inernational Journal of Human-Computer Interaction, 9 (2), pp. 183-193.

\section{Biography}

Sylnovie Merchant is an Assistant Professor of Information Systems at the College of Business Administration at California State University, Sacramento. She teaches both undergraduate and graduate courses in systems development. Her research interests include diffusion of technological innovations, social networks, human-computer interaction, and electronic commerce. Professor Merchant has published articles in a number of refereed journals and proceedings. 